\title{
FUNCTIONAL EQUATION FOR THE MORDELL-TORNHEIM MULTIPLE ZETA-FUNCTION
}

\author{
Takuya OKamoto, Tomokazu Onozuka
}

\begin{abstract}
We show a relation between the Mordell-Tornheim multiple zeta-function and the confluent hypergeometric function, and using it, we give the functional equation for the MordellTornheim multiple zeta-function. In the double case, the functional equation includes the known functional equation for the Euler-Zagier double zeta-function.
\end{abstract}

Keywords: Mordell-Tornheim multiple zeta-function, functional equation, confluent hypergeometric function.

\section{Introduction}

Let $r$ be a positive integer, $s_{j}$ be complex numbers for $j=1,2, \ldots, r+1$, and $i=\sqrt{-1}$ in this paper.

Matsumoto [8] introduced the Mordell-Tornheim multiple zeta-function

$$
\zeta_{M T, r}\left(s_{1}, \ldots, s_{r} ; s_{r+1}\right)=\sum_{m_{1}, \ldots, m_{r}=1}^{\infty} \frac{1}{m_{1}^{s_{1}} \cdots m_{r}^{s_{r}}\left(m_{1}+\cdots+m_{r}\right)^{s_{r+1}}}
$$

which is absolutely convergent in a certain region (see Lemma 2.1). In the case $r=2$, this series (1.1) was introduced by Tornheim [12] and he studied its values when these complex variables are integers in the region of absolute convergence. Also Mordell [10] independently considered the case $s_{1}=s_{2}=s_{3}$ of the above sum, and studied the values of the following multiple sum:

$$
\sum_{m_{1}, \ldots, m_{r}=1}^{\infty} m_{1}^{-1} \cdots m_{r}^{-1}\left(m_{1}+\cdots+m_{r}+a\right)^{-1}
$$

The first named author is supported by JSPS KAKENHI Grant Number 15K17517. The second named author is supported by JSPS KAKENHI Grant Number 13J00312.

2010 Mathematics Subject Classification: primary: 11M32; secondary: 11M06 
where $a>-r$. Also Matsumoto [8] proved that (1.1) can be continued meromorphically to $\mathbb{C}^{r+1}$ by the Mellin-Barnes integral formula and he determined the possible singularities of (1.1).

The major purpose of this paper is to study the functional equation for (1.1). In the case of the Euler-Zagier double zeta-function which is defined by

$$
\zeta_{E Z, 2}\left(s_{1}, s_{2}\right)=\sum_{m, n=1}^{\infty} \frac{1}{m^{s_{1}}(m+n)^{s_{2}}},
$$

the functional equation has been studied by Matsumoto [9]. We also note that (1.2) is absolutely convergent when $\Re s_{2}>1, \Re s_{1}+\Re s_{2}>2([8$, Theorem 3]) and can be continued meromorphically to $\mathbb{C}^{2}$ ([1, Theorem 1], [14, Theorem 5], [8]). In [9], Matsumoto gave the following functional equation for (1.2):

Theorem 1.1 ([9, Theorem 1]). We have

$$
\frac{g(u, v)}{(2 \pi)^{u+v-1} \Gamma(1-u)}=\frac{g(1-v, 1-u)}{i^{u+v-1} \Gamma(v)}+2 i \sin \left(\frac{\pi}{2}(u+v-1)\right) F_{+}(u, v),
$$

where $u, v$ are complex variables and $F_{+}(u, v), g(u, v)$ are defined by

$$
\begin{aligned}
F_{+}(u, v) & =\sum_{k=1}^{\infty} \sigma_{u+v-1}(k) \Psi(v, u+v ; 2 \pi i k), \\
g(u, v) & =\zeta_{E Z, 2}(u, v)-\frac{\Gamma(1-u)}{\Gamma(v)} \Gamma(u+v-1) \zeta(u+v-1),
\end{aligned}
$$

respectively. Also we put $\sigma_{l}(k)=\sum_{d \mid k} d^{l}$ and the confluent hypergeometric function

$$
\Psi(a, c ; x)=\frac{1}{\Gamma(a)} \int_{0}^{\infty e^{i \phi}} e^{-x y} y^{a-1}(1+y)^{c-a-1} d y
$$

where $\Re a>0,-\pi<\phi<\pi,|\phi+\arg x|<\pi / 2$.

The infinite series on the right-hand side of (1.4) is convergent only in the region $\Re u<0, \Re v>1$. However it can be continued meromorphically to $\mathbb{C}^{2}$ and satisfies a good functional equation ([9, Proposition 2]). The function $g(u, v)$ is a modified function of (1.2), and the equation (1.3) implies that $g(u, v)$ has a relation at two points $(u, v)$ and $(1-v, 1-u)$, thus the above theorem can be regarded as a functional equation for the Euler-Zagier double zeta-function. Furthermore, we have to note that Matsumoto gave the generalization of Theorem 1.1 ([9, Theorem 2]).

In the present paper, we discuss the same type of functional equation for the Mordell-Tornheim multiple zeta-function. We have to prepare some functions to state the main theorem. First we introduce two divisor functions

$$
\sigma_{a}\left(\ell_{1}, \ldots, \ell_{r}\right)=\sum_{d\left|\ell_{1}, \ldots, d\right| \ell_{r}} d^{a}
$$


and

$$
\sigma_{M T, r}\left(s_{1}, \ldots, s_{r}, s_{r+1} ; \ell_{1}, \ldots, \ell_{r}\right)=\sum_{d_{1}\left|\ell_{1}, \ldots, d_{r}\right| \ell_{r}} d_{1}^{s_{1}} \cdots d_{r}^{s_{r}}\left(d_{1}+\cdots+d_{r}\right)^{s_{r+1}},
$$

where $\ell_{1}, \ldots, \ell_{r}$ are positive integers and $a$ is a complex number. We call $\sigma_{a}$ and $\sigma_{M T, r}$ by $r$-divisor function and Mordell-Tornheim $r$-divisor function, respectively. These functions fulfill an important role in the present paper.

We also introduce two functions

$$
\begin{aligned}
F_{r}^{ \pm}\left(s_{1}, \ldots, s_{r+1}\right)= & \sum_{\ell_{1}, \ldots, \ell_{r-1}=1}^{\infty} \frac{\sigma_{s_{1}+\cdots+s_{r+1}-1}\left(\ell_{1}, \ldots, \ell_{r-1}\right)}{\ell_{1}^{s_{1}} \cdots \ell_{r-1}^{s_{r-1}}} \\
& \times \Psi\left(s_{r+1}, s_{r}+s_{r+1} ; \pm 2 \pi i\left(\ell_{1}+\cdots+\ell_{r-1}\right)\right)
\end{aligned}
$$

which performs the same role as (1.4) and this function is absolutely convergent when $\Re s_{j}>1, \Re s_{r}<0$ and $\Re s_{r+1}>0$ for $1 \leqslant j \leqslant r-1$ (see Theorem 3.3). Moreover, it can be continued meromorphically to $\mathbb{C}^{r+1}$ (see Theorem 3.4). Furthermore, we put

$$
\begin{aligned}
g_{r}\left(s_{1}, \ldots, s_{r+1}\right)= & \frac{1}{\prod_{j=1}^{r+1} \Gamma\left(s_{j}\right)} \int_{0}^{\infty} y^{s_{r+1}-1} \\
& \times\left(\prod_{j=1}^{r-1} f\left(s_{j}, y\right) \int_{0}^{\infty} x^{s_{r}-1} h\left(x_{r}+y\right) d x_{r}\right) d y
\end{aligned}
$$

where $f(s, y)=\int_{0}^{\infty} \frac{x^{s-1}}{e^{x+y}-1} d x$ and $h(z)=1 /\left(e^{z}-1\right)-1 / z$. This function performs the same role as (1.5) and can be continued meromorphically to the whole $\mathbb{C}^{r+1}$ space (see Theorem 3.4). Then we have the functional equation for (1.8).

\section{Theorem 1.2.}

We have

$$
\begin{aligned}
& \frac{g_{r}\left(-s_{1}, \ldots,-s_{r-1}, 1-s_{r+1}, 1-s_{r}\right)}{i^{s_{r}+s_{r+1}-1} \Gamma\left(s_{r+1}\right)} \\
& +e^{\frac{\pi i}{2}\left(s_{r}+s_{r+1}-1\right)} F_{r}^{+}\left(s_{1}, \ldots, s_{r+1}\right)+e^{-\frac{\pi i}{2}\left(s_{r}+s_{r+1}-1\right)} F_{r}^{-}\left(s_{1}, \ldots, s_{r+1}\right) \\
= & \frac{g_{r}\left(s_{1}, \ldots, s_{r-1}, s_{r}, s_{r+1}\right)}{(2 \pi)^{s_{r}+s_{r+1}-1} \Gamma\left(1-s_{r}\right)} \\
& +e^{-\frac{\pi i}{2}\left(s_{r}+s_{r+1}-1\right)} \sum_{\ell_{1}, \ldots, \ell_{r-1}=1}^{\infty} \sigma_{M T, r-1}\left(s_{1}, \ldots, s_{r-1}, s_{r}+s_{r+1}-1 ; \ell_{1}, \ldots, \ell_{r-1}\right) \\
& \times\left\{\Psi\left(s_{r+1}, s_{r}+s_{r+1} ; 2 \pi i\left(\ell_{1}+\cdots+\ell_{r-1}\right)\right)\right. \\
& \left.+\Psi\left(s_{r+1}, s_{r}+s_{r+1} ;-2 \pi i\left(\ell_{1}+\cdots+\ell_{r-1}\right)\right)\right\} .
\end{aligned}
$$


In Section 3, we will prove

$$
\begin{aligned}
& g_{r}\left(s_{1}, \ldots, s_{r+1}\right)=\zeta_{M T, r}\left(s_{1}, \ldots, s_{r} ; s_{r+1}\right) \\
& \quad-\frac{\Gamma\left(1-s_{r}\right) \Gamma\left(s_{r}+s_{r+1}-1\right)}{\Gamma\left(s_{r+1}\right)} \zeta_{M T, r-1}\left(s_{1}, \ldots, s_{r-1} ; s_{r}+s_{r+1}-1\right) .
\end{aligned}
$$

Hence, by (1.9), we are able to regard this theorem as the functional equation for the Mordell-Tornheim multiple zeta-function. Hence Theorem 1.2 is the main theorem in the present paper. Also, we note that Theorem 1.2 is a generalization of Theorem 1.1. Indeed, we will give the equation

$$
g_{2}\left(s_{1}, s_{2}, s_{3}\right)=\zeta_{M T, 2}\left(s_{1}, s_{2} ; s_{3}\right)-\frac{\Gamma\left(1-s_{2}\right) \Gamma\left(s_{2}+s_{3}-1\right)}{\Gamma\left(s_{3}\right)} \zeta_{M T, 1}\left(s_{1} ; s_{2}+s_{3}-1\right)
$$

by (1.9). Hence we have

$$
g_{2}\left(0, s_{1}, s_{2}\right)=g\left(s_{1}, s_{2}\right)
$$

since $\zeta_{M T, 2}\left(0, s_{1} ; s_{2}\right)=\zeta_{E Z, 2}\left(s_{1}, s_{2}\right)$ and $\zeta_{M T, 1}\left(0 ; s_{1}+s_{2}-1\right)=\zeta\left(s_{1}+s_{2}-1\right)$. Moreover, we have

$$
F_{2}^{ \pm}\left(0, s_{1}, s_{2}\right)=\sum_{\ell=1}^{\infty} \sigma_{s_{1}+s_{2}-1}(\ell) \Psi\left(s_{2}, s_{1}+s_{2} ; \pm 2 \pi i \ell\right)
$$

and

$$
\sigma_{M T, 1}\left(0, s_{1}+s_{2}-1 ; \ell\right)=\sigma_{s_{1}+s_{2}-1}(\ell) .
$$

Substituting (1.10), (1.11) and (1.12) to Theorem 1.2, we obtain Theorem 1.1.

We have to study (1.8) and show the relation between the Mordell-Tornheim multiple zeta-function and the confluent hypergeometric function (see Theorem 3.3) to prove Theorem 1.2. Moreover, to show the relation, we have to use the residue of calculus around the contour $C(R)$ and $D(R)$ (see proof of Theorem 3.2). The same type of the residue calculus in the present paper has been often applied to study the functional equation (e.g., [3], [6], [7] and [9]).

Also (1.1) relates with the zeta-function of the root system of type $A_{r}$ defined by

$$
\zeta_{r}\left(\mathbf{s} ; A_{r}\right)=\sum_{m_{1}, \ldots, m_{r}=1}^{\infty} \prod_{1 \leqslant i<j \leqslant r+1}\left(m_{i}+\cdots+m_{j-1}\right)^{-s_{i j}}
$$

where $\mathbf{s}=\left(s_{i j}\right) \in \mathbb{C}^{r(r+1) / 2}$. In fact, we have

$$
\zeta_{r}\left(\mathbf{s} ; A_{r}\right)=\zeta_{M T, r}\left(s_{12}, \ldots, s_{r, r+1} ; s_{1, r+1}\right),
$$


where $\mathbf{s}=\left(s_{12}, s_{23}, s_{34}, \ldots, s_{r, r+1}, s_{1, r+1}, 0, \ldots, 0\right)$. Especially, the zeta-function of the root system of type $A_{2}$ is identical with the Mordell-Tornheim double zetafunction. The zeta-function of the root system is introduced by Komori, Matsumoto and Tsumura [5] and this function is a generalization of the Witten zetafunction introduced by Witten [13] which is an important function in mathematical physics. Hence it is valuable that we study the functional equation for (1.1) in this viewpoint.

In the next section, we give two lemmas to prove Theorem 3.4 which implies that $g_{r}\left(s_{1}, \ldots, s_{r+1}\right)$ can be continued meromorphically to the whole $\mathbb{C}^{r+1}$. Theorem 3.4 is one of the most important theorem to prove Theorem 1.2.

\section{The region of absolute convergence and convolution for $\zeta_{M T, r}$}

First we give the region of absolute convergence for (1.1). This is the generalization of Theorem 2.2 in [11].

Lemma 2.1. The series (1.1) is absolutely convergent when

$$
\sum_{\ell=1}^{j} \Re s_{k_{\ell}}+\Re s_{r+1}>j
$$

with $1 \leqslant k_{1}<k_{2}<\cdots<k_{j} \leqslant r$ for any $j=1,2, \ldots, r$.

Proof. We prove this theorem by induction. In the case $r=2$, this theorem is Theorem 2.2 in [11]. Hence we assume the validity of Lemma 2.1 in the case $r=k$, and we will prove Lemma 2.1 in the case $r=k+1$. By Lemma 2.1 in [11] as $n=m_{k+1}$ and $m=m_{1}+\cdots+m_{k}$, we have

$$
\begin{aligned}
& \left|\zeta_{M T, k+1}\left(s_{1}, \ldots, s_{k+1} ; s_{k+2}\right)\right| \\
& \ll \sum_{m_{1}, \ldots, m_{k}=1}^{\infty} \frac{1}{m_{1}^{\Re s_{1}} \cdots m_{k}^{\Re s_{k}}} \sum_{m_{k+1}=1}^{\infty} \frac{1}{m_{k+1}^{\Re s_{k+1}}\left(m_{1}+\cdots+m_{k+1}\right)^{\Re s_{k+2}}} \\
& \quad \ll \begin{cases}\sum_{m_{1}, \ldots, m_{k}=1}^{\infty} \frac{1}{m_{1}^{\Re s_{1}} \cdots m_{k}^{\Re s_{k}}\left(m_{1}+\cdots+m_{k}\right)^{\Re s_{k+2}}} & \left(\Re s_{k+1}>1\right) \\
\sum_{m_{1}, \ldots, m_{k}=1}^{\infty} \frac{\log \left(m_{1}+\cdots+m_{k}\right)}{m_{1}^{\Re s_{1}} \cdots m_{k}^{\Re s_{k}}\left(m_{1}+\cdots+m_{k}\right)^{\Re s_{k+2}}} & \left(\Re s_{k+1}=1\right) \\
\sum_{m_{1}, \ldots, m_{k}=1}^{\infty} \frac{1}{m_{1}^{\Re s_{1}} \cdots m_{k}^{\Re s_{k}}\left(m_{1}+\cdots+m_{k}\right)^{\Re s_{k+1}+\Re s_{k+2}-1}} & (\Re) .\end{cases}
\end{aligned}
$$

By the assumption of Lemma 2.1 in the case $r=k$, we obtain Lemma 2.1 when $r=k+1$. 
Next we introduce a convolution of the multiple zeta-function. We put

$$
\zeta_{M T, r}\left(s_{1}, \ldots, s_{r} ; s_{r+1} ; f\right)=\sum_{m_{1}, \ldots, m_{r}=1}^{\infty} \frac{f\left(m_{1}, \ldots, m_{r}\right)}{m_{1}^{s_{1}} \cdots m_{r}^{s_{r}}\left(m_{1}+\cdots+m_{r}\right)^{s_{r+1}}}
$$

where $f$ is a multi-variable arithmetic function.

Lemma 2.2. Let a be a complex number. Then, in the region

$$
\begin{array}{ll}
\Re s_{k_{1}}+\Re s_{r+1}>1 & \left(1 \leqslant k_{1} \leqslant r\right), \\
\Re s_{k_{1}}+\Re s_{k_{2}}+\Re s_{r+1}>2 & \left(1 \leqslant k_{1}<k_{2} \leqslant r\right), \\
\quad \ldots & \left(1 \leqslant k_{1}<k_{2}<\cdots<k_{r-1} \leqslant r\right), \\
\Re s_{k_{1}}+\Re s_{k_{2}}+\cdots+\Re s_{k_{r-1}}+\Re s_{r+1}>r-1 & \\
\Re s_{1}+\Re s_{2}+\cdots+\Re s_{r+1}>\max \{r, \Re a+1\}, &
\end{array}
$$

we have

$$
\zeta(s-a) \zeta_{M T, r}\left(s_{1}, \ldots, s_{r} ; s_{r+1}\right)=\zeta_{M T, r}\left(s_{1}, \ldots, s_{r} ; s_{r+1} ; \sigma_{a}\left(m_{1}, \ldots, m_{r}\right)\right),
$$

where $s=s_{1}+\cdots+s_{r+1}$.

Proof. In the above region, $\zeta_{M T, r}\left(s_{1}, \ldots, s_{r} ; s_{r+1}\right)$ and $\zeta(s-a)$ are absolutely convergent by Lemma 2.1. Hence we have

$$
\begin{aligned}
\zeta(s-a) \zeta_{M T, r}\left(s_{1}, \ldots, s_{r} ; s_{r+1}\right) & \\
& =\sum_{n, m_{1}, \ldots, m_{r}=1}^{\infty} \frac{n^{a}}{\left(n m_{1}\right)^{s_{1}} \cdots\left(n m_{r}\right)^{s_{r}}\left(n m_{1}+\cdots+n m_{r}\right)^{s_{r+1}}} \\
& =\sum_{\ell_{1}, \ldots, \ell_{r}=1}^{\infty} \frac{\sum_{n\left|\ell_{1}, \ldots, n\right| \ell_{r}} n^{a}}{\ell_{1}^{s_{1}} \cdots \ell_{r}^{s_{r}}\left(\ell_{1}+\cdots+\ell_{r}\right)^{s_{r+1}}} .
\end{aligned}
$$

Therefore we obtain this lemma.

\section{Integral expression for $\zeta_{M T, r}$ and the properties of $g_{r}$}

We give the following integral expression for (1.1).

Theorem 3.1. In the region $\Re s_{j}>1$ and $\Re s_{r+1}>0$ for $1 \leqslant j \leqslant r$, we have

$$
\zeta_{M T, r}\left(s_{1}, \ldots, s_{r} ; s_{r+1}\right)=\frac{1}{\prod_{j=1}^{r+1} \Gamma\left(s_{j}\right)} \int_{0}^{\infty} y^{s_{r+1}-1}\left(\prod_{j=1}^{r} f\left(s_{j}, y\right)\right) d y
$$

where $f(s, y)=\int_{0}^{\infty} \frac{x^{s-1}}{e^{x+y}-1} d x$ for $y \geqslant 0$ and $s \in \mathbb{C}$ with $\Re s>1$. 
Proof. First we assume $\Re s_{j}>1$ and $\Re s_{r+1}>0$ for $1 \leqslant j \leqslant r$. For $1 \leqslant j \leqslant r$, we have

$$
\Gamma\left(s_{j}\right) \sum_{m_{j}=1}^{\infty} e^{-m_{j} y} m_{j}^{-s_{j}}=f\left(s_{j}, y\right) .
$$

By (3.2), we have

$$
\begin{aligned}
\prod_{j=1}^{r+1} \Gamma\left(s_{j}\right) \sum_{m_{1}, \ldots, m_{r}=1}^{\infty} m_{1}^{-s_{1}} \cdots m_{r}^{-s_{r}}\left(m_{1}+\cdots\right. & \left.+m_{r}\right)^{-s_{r+1}} \\
& =\int_{0}^{\infty} y^{s_{r+1}-1}\left(\prod_{j=1}^{r} f\left(s_{j}, y\right)\right) d y
\end{aligned}
$$

This equality is justified since $\Re s_{j}>1$ and $\Re s_{r+1}>0$ for $1 \leqslant j \leqslant r$. Hence we obtain Theorem 3.1.

Next, using the function $h(z)=1 /\left(e^{z}-1\right)-1 / z$, we divide the right-hand side of (3.1) in order to deform the path of the integral. We have

$$
\begin{aligned}
& \zeta_{M T, r}\left(s_{1}, \ldots, s_{r} ; s_{r+1}\right) \\
&= \frac{1}{\prod_{j=1}^{r+1} \Gamma\left(s_{j}\right)} \int_{0}^{\infty} y^{s_{r+1}-1}\left(\prod_{j=1}^{r-1} f\left(s_{j}, y\right) \int_{0}^{\infty} x_{r}^{s_{r}-1} h\left(x_{r}+y\right) d x_{r}\right) d y \\
&+\frac{1}{\prod_{j=1}^{r+1} \Gamma\left(s_{j}\right)} \int_{0}^{\infty} y^{s_{r+1}-1}\left(\prod_{j=1}^{r-1} f\left(s_{j}, y\right) \int_{0}^{\infty} \frac{x_{r}^{s_{r}-1}}{x_{r}+y} d x_{r}\right) d y \\
&= g_{r}\left(s_{1}, \ldots, s_{r+1}\right)+g_{r}^{*}\left(s_{1}, \ldots, s_{r+1}\right),
\end{aligned}
$$

say. Using (3.1) and the reflection formula

$$
\int_{0}^{\infty} \frac{x_{r}^{s_{r}-1}}{x_{r}+y} d x_{r}=y^{s_{r}-1} \Gamma\left(s_{r}\right) \Gamma\left(1-s_{r}\right),
$$

we have

$$
\begin{aligned}
g_{r}^{*}\left(s_{1}, \ldots, s_{r+1}\right)= & \frac{\Gamma\left(1-s_{r}\right)}{\Gamma\left(s_{r+1}\right) \prod_{j=1}^{r-1} \Gamma\left(s_{j}\right)} \int_{0}^{\infty} y^{s_{r}+s_{r+1}-2}\left(\prod_{j=1}^{r-1} f\left(s_{j}, y\right)\right) d y \\
= & \frac{\Gamma\left(1-s_{r}\right) \Gamma\left(s_{r}+s_{r+1}-1\right)}{\Gamma\left(s_{r+1}\right)} \\
& \times \zeta_{M T, r-1}\left(s_{1}, \ldots, s_{r-1} ; s_{r}+s_{r+1}-1\right)
\end{aligned}
$$

Substituting (3.4) to (3.3), we obtain (1.9). 
Now we start to consider the functional equation for (1.8). We first deform the path to the contour $C$, which consists of the half-line on the positive real axis from infinity to a small positive number, a small circle counterclockwise round the origin and the other half-line on the positive real axis back to infinity. We also remark that $\Im \log x_{r}$ varies from 0 to $2 \pi$ round $C$. Hence we have

$$
\begin{aligned}
g_{r}\left(s_{1}, \ldots, s_{r+1}\right)= & \frac{1}{\left(e^{2 \pi i s_{r}}-1\right) \prod_{j=1}^{r+1} \Gamma\left(s_{j}\right)} \\
& \times \int_{0}^{\infty} y^{s_{r+1}-1} \int_{C} x_{r}^{s_{r}-1} h\left(x_{r}+y\right) d x_{r}\left(\prod_{j=1}^{r-1} f\left(s_{j}, y\right)\right) d y .
\end{aligned}
$$

The right-hand side of the above equality is absolutely convergent when $\Re s_{r+1}>0$, $\Re s_{r}<1$ and $\Re s_{j}>1$ for $1 \leqslant j \leqslant r-1$. In fact, by the following estimate in [4],

$$
h(x+y)=O\left(e^{-K|x|}+(|x|+1)^{-1}\right),
$$

which holds with a positive absolute constant $K$, uniformly for any $x, y \in C \cup$ $[0, \infty)$, we obtain

$$
\int_{C} x_{r}^{s_{r}-1} h\left(x_{r}+y\right) d x_{r}=O(1)
$$

when $\Re s_{r}<1$. Also, for $\Re s>1$, we have

$$
f(s, 0)=O(1)
$$

since

$$
\frac{1}{e^{x}-1} \ll \begin{cases}x^{-1} & \text { if } 0<x \leqslant 2 \\ e^{-x} & \text { if } x \geqslant 2\end{cases}
$$
have

Then, in the region $\Re s_{r+1}>0, \Re s_{r}<1$ and $\Re s_{j}>1$ for $1 \leqslant j \leqslant r-1$, we

$$
g_{r}\left(s_{1}, \ldots, s_{r+1}\right) \ll \int_{0}^{\infty} y^{\Re s_{r+1}-1} f\left(\Re s_{1}, y\right) d y \ll 1
$$

where the first estimate is justified by (3.6) and (3.7), and the second follows from

$$
f(s, y) \ll \begin{cases}1 & \text { if } 0<y \leqslant 2 \\ e^{-y} & \text { if } y \geqslant 2\end{cases}
$$

for $\Re s>1$. 
Next, changing the path of the integral in $x_{r}$, we obtain the following theorem:

Theorem 3.2. In the region $\Re s_{j}>1, \Re s_{r}<0$ and $\Re s_{r+1}>0$ for $1 \leqslant j \leqslant r-1$, we have

$$
g_{r}\left(s_{1}, \ldots, s_{r+1}\right)=\frac{-2 \pi i}{\left(e^{2 \pi i s_{r}}-1\right) \prod_{j=1}^{r+1} \Gamma\left(s_{j}\right)} \sum_{n \neq 0} I_{r}(n),
$$

where

$$
I_{r}(n)=\int_{0}^{\infty} y^{s_{r+1}-1}(-y+2 \pi i n)^{s_{r}-1}\left(\prod_{j=1}^{r-1} f\left(s_{j}, y\right)\right) d y
$$

Proof. First, by the residue theorem, we obtain

$$
\begin{aligned}
& \int_{0}^{R / 2} y^{s_{r+1}-1} \int_{C(R)} x_{r}^{s_{r}-1} h\left(x_{r}+y\right) d x_{r}\left(\prod_{j=1}^{r-1} f\left(s_{j}, y\right)\right) d y \\
+ & \int_{0}^{R / 2} y^{s_{r+1}-1} \int_{D(R)} x_{r}^{s_{r}-1} h\left(x_{r}+y\right) d x_{r}\left(\prod_{j=1}^{r-1} f\left(s_{j}, y\right)\right) d y \\
= & -2 \pi i \int_{0}^{R / 2} y^{s_{r+1}-1} \sum_{|n| \leqslant N, n \neq 0}(-y+2 \pi i n)^{s_{r}-1}\left(\prod_{j=1}^{r-1} f\left(s_{j}, y\right)\right) d y
\end{aligned}
$$

where $R=2 \pi(N+1 / 2)$ for a sufficiently large positive integer $N$, the contour $C(R)$ consists of the half-line on the positive real axis from $-y+R$ to a small positive number, a small circle counterclockwise round the origin and the other half-line on the positive real axis back to $-y+R$ and the contour $D(R)$ consists of a circle of the radius $R$ clockwise round $-y$.

By the above argument, we have

$$
\begin{aligned}
& \int_{0}^{R / 2} y^{s_{r+1}-1} \int_{C(R)} x_{r}^{s_{r}-1} h\left(x_{r}+y\right) d x_{r}\left(\prod_{j=1}^{r-1} f\left(s_{j}, y\right)\right) d y \\
& \rightarrow \int_{0}^{\infty} y^{s_{r+1}-1} \int_{C} x_{r}^{s_{r}-1} h\left(x_{r}+y\right) d x_{r}\left(\prod_{j=1}^{r-1} f\left(s_{j}, y\right)\right) d y \quad(R \rightarrow \infty)
\end{aligned}
$$

in the region $\Re s_{j}>1, \Re s_{r}<1$ and $\Re s_{r+1}>0$ for $1 \leqslant j \leqslant r-1$. 
Next we estimate the second term on the left-hand side of (3.11). By (3.7) and (3.8), we have

$$
\begin{aligned}
\int_{0}^{R / 2} y^{s_{r+1}-1} \int_{D(R)} x_{r}^{s_{r}-1} h\left(x_{r}+y\right) d x_{r}\left(\prod_{j=1}^{r-1} f\left(s_{j}, y\right)\right) d y \\
\ll R^{\Re s_{r}}\left(\int_{0}^{2} y^{\Re s_{r+1}-1} d y+\int_{2}^{R / 2} y^{\Re s_{r+1}-1} e^{-y} d y\right) \\
\ll R^{\Re s_{r}} \rightarrow 0 \quad(R \rightarrow \infty)
\end{aligned}
$$

in the region $\Re s_{j}>1, \Re s_{r}<0$ and $\Re s_{r+1}>0$ for $1 \leqslant j \leqslant r-1$.

Lastly we consider the right-hand side of (3.11). In the region $\Re s_{j}>1, \Re s_{r}<0$ and $\Re s_{r+1}>0$ for $1 \leqslant j \leqslant r-1$, we obtain

$$
\begin{aligned}
\int_{0}^{\infty} y^{s_{r+1}-1} \sum_{n \neq 0}(-y+2 \pi i n)^{s_{r}-1}\left(\prod_{j=1}^{r-1} f\left(s_{j}, y\right)\right) d y \\
\ll \int_{0}^{2} y^{\Re s_{r+1}-1} \sum_{n=1}^{\infty}|-y+2 \pi i n|^{\Re s_{r}-1} d y \\
\quad+\int_{2}^{\infty} y^{\Re s_{r+1}-1} \sum_{n=1}^{\infty} e^{-y}|-y+2 \pi i n|^{\Re s_{r}-1} d y \\
\ll \int_{0}^{2} y^{\Re s_{r+1}-1} d y \sum_{n=1}^{\infty} n^{\Re s_{r}-1}+\int_{2}^{\infty} y^{\Re s_{r+1}-1} e^{-y} d y \sum_{n=1}^{\infty} n^{\Re s_{r}-1} \ll 1
\end{aligned}
$$

by (3.7) and (3.8). Hence we have

$$
\begin{aligned}
& -2 \pi i \int_{0}^{R / 2} y^{s_{r+1}-1} \sum_{|n| \leqslant N, n \neq 0}(-y+2 \pi i n)^{s_{r}-1}\left(\prod_{j=1}^{r-1} f\left(s_{j}, y\right)\right) d y \\
& \rightarrow-2 \pi i \int_{0}^{\infty} y^{s_{r+1}-1} \sum_{n \neq 0}(-y+2 \pi i n)^{s_{r}-1}\left(\prod_{j=1}^{r-1} f\left(s_{j}, y\right)\right) d y \quad(R \rightarrow \infty)
\end{aligned}
$$

in the region $\Re s_{j}>1, \Re s_{r}<0$ and $\Re s_{r+1}>0$ for $1 \leqslant j \leqslant r-1$.

Therefore we obtain Theorem 3.2 by (3.12), (3.13) and (3.15).

Now we assume $\Re s_{j}>1, \Re s_{r}<0$ and $\Re s_{r+1}>0$ for $1 \leqslant j \leqslant r-1$. Then, in the case $n>0$, by (3.2) and changing the variable of the integral for $I_{r}(n)$ by $y=e^{-\pi i / 2} 2 \pi n y^{\prime}$, we obtain by using (1.6) with $x=2 \pi n\left(m_{1}+\cdots+m_{r-1}\right) e^{-\pi i / 2}$ 
and $\phi=\pi / 2$ that

$$
\begin{aligned}
\sum_{n>0} I_{r}(n)= & \sum_{n=1}^{\infty}\left(\prod_{j=1}^{r-1} \Gamma\left(s_{j}\right)\right) \sum_{m_{1}, \ldots, m_{r-1}=1}^{\infty} m_{1}^{-s_{1}} \cdots m_{r-1}^{-s_{r-1}} \\
& \times \int_{0}^{\infty} y^{s_{r+1}-1}(-y+2 \pi i n)^{s_{r}-1} e^{-\left(m_{1}+\cdots+m_{r-1}\right) y} d y \\
= & (2 \pi)^{s_{r}+s_{r+1}-1} e^{\pi i\left(s_{r}-s_{r+1}-1\right) / 2}\left(\prod_{j=1}^{r-1} \Gamma\left(s_{j}\right)\right) \\
& \times \Gamma\left(s_{r+1}\right) \sum_{m_{1}, \ldots, m_{r-1}=1}^{\infty} m_{1}^{-s_{1}} \cdots m_{r-1}^{-s_{r-1}} \\
& \times \sum_{n=1}^{\infty} n^{s_{r}+s_{r+1}-1} \Psi\left(s_{r+1}, s_{r}+s_{r+1} ;-2 \pi i n\left(m_{1}+\cdots+m_{r-1}\right)\right) .
\end{aligned}
$$

Similarly, in the case $n<0$, changing the variable $y=2 \pi e^{\pi i / 2}|n| y^{\prime}$ in the integral for $I_{r}(n)$ and using (1.6) with $x=2 \pi|n|\left(m_{1}+\cdots+m_{r-1}\right) e^{\pi i / 2}$ and $\phi=-\pi / 2$ we obtain

$$
\begin{aligned}
\sum_{n<0} I_{r}(n)= & -(2 \pi)^{s_{r}+s_{r+1}-1} e^{\pi i\left(3 s_{r}+s_{r+1}-1\right) / 2}\left(\prod_{j=1}^{r-1} \Gamma\left(s_{j}\right)\right) \\
& \times \Gamma\left(s_{r+1}\right) \sum_{m_{1}, \ldots, m_{r-1}=1}^{\infty} m_{1}^{-s_{1}} \cdots m_{r-1}^{-s_{r-1}} \\
& \times \sum_{n=1}^{\infty} n^{s_{r}+s_{r+1}-1} \Psi\left(s_{r+1}, s_{r}+s_{r+1} ; 2 \pi i n\left(m_{1}+\cdots+m_{r-1}\right)\right) .
\end{aligned}
$$

Hence, using the formula

$$
\frac{1}{\Gamma\left(s_{r}\right)\left(e^{2 \pi i s_{r}}-1\right)}=\frac{\Gamma\left(1-s_{r}\right)}{2 \pi i e^{\pi i s_{r}}}
$$

we obtain

$$
\begin{aligned}
g_{r}\left(s_{1}, \ldots, s_{r+1}\right)= & (2 \pi)^{s_{r}+s_{r+1}-1} \Gamma\left(1-s_{r}\right) \sum_{m_{1}, \ldots, m_{r-1}=1}^{\infty} m_{1}^{-s_{1}} \cdots m_{r-1}^{-s_{r-1}} \\
& \times\left\{e^{-\pi i\left(s_{r}+s_{r+1}-1\right) / 2} \sum_{n=1}^{\infty} n^{s_{r}+s_{r+1}-1}\right. \\
& \times \Psi\left(s_{r+1}, s_{r}+s_{r+1} ;-2 \pi i n\left(m_{1}+\cdots+m_{r-1}\right)\right) \\
& +e^{\pi i\left(s_{r}+s_{r+1}-1\right) / 2} \sum_{n=1}^{\infty} n^{s_{r}+s_{r+1}-1} \\
& \left.\times \Psi\left(s_{r+1}, s_{r}+s_{r+1} ; 2 \pi i n\left(m_{1}+\cdots+m_{r-1}\right)\right)\right\} .
\end{aligned}
$$


We remark that the definition of the confluent hypergeometric function $\Psi$ is given by (1.6). Then, putting $\ell_{j}=m_{j} n$ and changing the sum on the right-hand side of (3.16), we can express (1.8) in terms of (1.7):

Theorem 3.3. In the region $\Re s_{j}>1, \Re s_{r}<0$ and $\Re s_{r+1}>0$ for $1 \leqslant j \leqslant r-1$, we have

$$
\begin{aligned}
& g_{r}\left(s_{1}, \ldots, s_{r+1}\right)=(2 \pi)^{s_{r}+s_{r+1}-1} \Gamma\left(1-s_{r}\right) \\
& \quad \times\left(e^{\pi i\left(s_{r}+s_{r+1}-1\right) / 2} F_{r}^{+}\left(s_{1}, \ldots, s_{r+1}\right)+e^{-\pi i\left(s_{r}+s_{r+1}-1\right) / 2} F_{r}^{-}\left(s_{1}, \ldots, s_{r+1}\right)\right) .
\end{aligned}
$$

Recalling (1.7) and (1.9), Theorem 3.3 shows the relation between the MordellTornheim multiple zeta-function and the confluent hypergeometric function. Now we have to consider the analytic continuation for (1.8) to give the functional equation for (1.8).

Theorem 3.4. The functions $F_{r}^{ \pm}\left(s_{1}, \ldots, s_{r+1}\right)$ and $g_{r}\left(s_{1}, \ldots, s_{r+1}\right)$ can be continued meromorphically to the whole space $\mathbb{C}^{r+1}$.

Note that the region where Theorem 3.3 is valid can be extended to the whole space by Theorem 3.4 .

Proof of Theorem 3.4. First we assume $\Re s_{j}>1, \Re s_{r}<0$ and $\Re s_{r+1}>0$ for $1 \leqslant j \leqslant r-1$. Then, by Theorem 3.3, it is sufficient to show that $F_{r}^{ \pm}$can be continued meromorphically to the whole $\mathbb{C}^{r+1}$.

Applying the formula (cf. [2, $6.5(6)]$ )

$$
\Psi(a, c ; x)=x^{1-c} \Psi(a-c+1,2-c ; x)
$$

to $(1.7)$, we obtain

$$
\begin{aligned}
& F_{r}^{ \pm}\left(s_{1}, \ldots, s_{r+1}\right) \\
& =( \pm 2 \pi i)^{1-s_{r}-s_{r+1}} \sum_{\ell_{1}, \ldots, \ell_{r-1}=1}^{\infty} \frac{\sigma_{s_{1}+\cdots+s_{r+1}-1}\left(\ell_{1}, \ldots, \ell_{r-1}\right)}{\ell_{1}^{s_{1}} \cdots \ell_{r-1}^{s_{r}-1}\left(\ell_{1}+\cdots+\ell_{r-1}\right)^{s_{r}+s_{r+1}-1}} \\
& \quad \times \Psi\left(1-s_{r}, 2-s_{r}-s_{r+1} ; \pm 2 \pi i\left(\ell_{1}+\cdots+\ell_{r-1}\right)\right)
\end{aligned}
$$

We remark that we have the asymptotic expansion (cf. [2, 6.13.1 (1)])

$$
\Psi(a, c ; x)=\sum_{k=0}^{N-1} \frac{(-1)^{k}(a-c+1)_{k}(a)_{k}}{k !} x^{-a-k}+\rho_{N}(a, c ; x),
$$

where $N$ is an arbitrary non-negative integer, $(a)_{k}=\Gamma(a+k) / \Gamma(k)$ and

$$
\begin{aligned}
& \rho_{N}(a, c ; x) \\
= & \frac{(-1)^{N}(a-c+1)_{N}}{\Gamma(a)} \int_{0}^{\infty e^{i \phi}} e^{-x y} y^{a+N-1} \int_{0}^{1} \frac{(1-\tau)^{N-1}}{(N-1) !}(1+\tau y)^{c-a-N-1} d \tau d y .
\end{aligned}
$$


Applying (3.20) to (3.19) and using Lemma 2.2, we obtain

$$
\begin{aligned}
F_{r}^{ \pm}\left(s_{1}, \ldots, s_{r+1}\right) & \\
= & \sum_{k=0}^{N-1} \frac{(-1)^{k}\left(s_{r+1}\right)_{k}\left(1-s_{r}\right)_{k}}{( \pm 2 \pi i)^{s_{r+1}+k} k !} \zeta_{M T, r-1}\left(s_{1}, \ldots, s_{r-1} ; s_{r+1}+k\right) \zeta\left(-s_{r}+1+k\right) \\
& +( \pm 2 \pi i)^{1-s_{r}-s_{r+1}} \sum_{\ell_{1}, \ldots, \ell_{r-1}=1}^{\infty} \frac{\sigma_{s_{1}+\cdots+s_{r+1}-1}\left(\ell_{1}, \ldots, \ell_{r-1}\right)}{\ell_{1}^{s_{1}} \cdots \ell_{r-1}^{s_{r-1}}\left(\ell_{1}+\cdots+\ell_{r-1}\right)^{s_{r}+s_{r+1}-1}} \\
& \times \rho_{N}\left(1-s_{r}, 2-s_{r}-s_{r+1} ; \pm 2 \pi i\left(\ell_{1}+\cdots+\ell_{r-1}\right)\right) .
\end{aligned}
$$

Applying the estimate (cf. [7, (6.2)])

$$
\begin{aligned}
& \left|\rho_{N}\left(1-s_{r}, 2-s_{r}-s_{r+1} ; \pm 2 \pi i\left(\ell_{1}+\cdots+\ell_{r-1}\right)\right)\right| \\
& \leqslant \frac{\left|\left(s_{r+1}\right)_{N}\right| \Gamma\left(-\Re s_{r}+N+1\right)}{N !\left|\Gamma\left(1-s_{r}\right)\right|} e^{\pi\left(\left|\Im s_{r}\right|+\left|\Im s_{r+1}\right|\right) / 2}\left(2 \pi\left(\ell_{1}+\cdots+\ell_{r-1}\right)\right)^{\Re s_{r}-N-1},
\end{aligned}
$$

where $\Re s_{r}<N+1$ and $\Re s_{r+1} \geqslant-N$, to the second term on the right-hand side of (3.21) and using Lemma 2.2, we have

$$
\begin{aligned}
& ( \pm 2 \pi i)^{1-s_{r}-s_{r+1}} \sum_{\ell_{1}, \ldots, \ell_{r-1}=1}^{\infty} \frac{\sigma_{s_{1}+\cdots+s_{r+1}-1}\left(\ell_{1}, \ldots, \ell_{r-1}\right)}{\ell_{1}^{s_{1}} \cdots \ell_{r-1}^{s_{r}-1}\left(\ell_{1}+\cdots+\ell_{r-1}\right)^{s_{r}+s_{r+1}-1}} \\
\times & \rho_{N}\left(1-s_{r}, 2-s_{r}-s_{r+1} ; \pm 2 \pi i\left(\ell_{1}+\cdots+\ell_{r-1}\right)\right) \\
\ll & \frac{\left|\left(s_{r+1}\right)_{N}\right| \Gamma\left(-\Re s_{r}+N+1\right)}{N !\left|\Gamma\left(1-s_{r}\right)\right|} e^{\pi\left(\left|\Im s_{r}\right|+\left|\Im s_{r+1}\right|\right)} \\
& \times \zeta_{M T, r-1}\left(\Re s_{1}, \ldots, \Re s_{r-1} ; \Re s_{r+1}+N\right) \zeta\left(-\Re s_{r}+1+N\right) .
\end{aligned}
$$

Hence the second term on the right-hand side of (3.21) is convergent absolutely when $\Re s_{r}<N$ and

$$
\sum_{\ell=1}^{j} \Re s_{k_{\ell}}+\Re s_{r+1}>j-N
$$

with $1 \leqslant k_{1}<k_{2}<\cdots<k_{j} \leqslant r-1$ for any $j=1,2, \ldots, r-1$ by Lemma 2.1 . Since $N$ is arbitrary, $F_{r}^{ \pm}\left(s_{1}, \ldots, s_{r+1}\right)$ can be continued meromorphically to the whole space $\mathbb{C}^{r+1}$.

Lastly we give the proof of Theorem 1.2.

Proof of Theorem 1.2. . By Theorem 3.4, $g_{r}\left(s_{1}, \ldots, s_{r+1}\right)$ can be continued meromorphically to the whole $\mathbb{C}^{r+1}$. Hence, changing $\left(s_{1}, \ldots, s_{r-1}, s_{r}, s_{r+1}\right)$ by $\left(-s_{1}, \ldots,-s_{r-1}, 1-s_{r+1}, 1-s_{r}\right)$ in (3.19) and then rearranging the $l_{j}$ sum on 
putting $l_{j}=d m_{j}(j=1,2, \ldots, r-1)$, we have

$$
\begin{aligned}
& g_{r}\left(-s_{1}, \ldots,-s_{r-1}, 1-s_{r+1}, 1-s_{r}\right) \\
&= \Gamma\left(s_{r+1}\right) \times\left\{\sum_{\ell_{1}, \ldots, \ell_{r-1}=1}^{\infty} \sigma_{M T, r-1}\left(s_{1}, \ldots, s_{r-1}, s_{r}+s_{r+1}-1 ; \ell_{1}, \ldots, \ell_{r-1}\right)\right. \\
& \times \Psi\left(s_{r+1}, s_{r}+s_{r+1} ;-2 \pi i\left(\ell_{1}+\cdots+\ell_{r-1}\right)\right) \\
&+\sum_{\ell_{1}, \ldots, \ell_{r-1}=1}^{\infty} \sigma_{M T, r-1}\left(s_{1}, \ldots, s_{r-1}, s_{r}+s_{r+1}-1 ; \ell_{1}, \ldots, \ell_{r-1}\right) \\
&\left.\times \Psi\left(s_{r+1}, s_{r}+s_{r+1} ; 2 \pi i\left(\ell_{1}+\cdots+\ell_{r-1}\right)\right)\right\} .
\end{aligned}
$$

By (3.17) and (3.22), we obtain Theorem 1.2.

\section{References}

[1] S. Akiyama, S. Egami and Y. Tanigawa, Analytic continuation of multiple zeta-functions and their values at non-positive integers, Acta Arith. 98 (2001), $107-116$.

[2] A. Erdélyi et al, (the Bateman Manuscript Project), Higher transendental functions, vol. I, (McGraw-Hill, 1953).

[3] M. Katsurada and K. Matsumoto, Asymptotic expansions of the mean values of Dirichlet L-functions, Math. Z. 208 (1991), 23-39.

[4] M. Katsurada and K. Matsumoto, Explicit formulas and asymptotic expansions for certain mean square of Hurwitz zeta-functions, Math. Scand. 78 (1996), 161-177.

[5] Y. Komori, K. Matsumoto and H. Tsumura, On Witten multiple zetafunctions associated with semisimple Lie algebras II, J. Math. Soc. Japan 62 (2010), no. 2, 355-394.

[6] Y. Komori, K. Matsumoto and H. Tsumura, Functional equations for double L-functions and values at non-positive integers, Int. J. Number Theory 7 (2011), 1441-1461.

[7] K. Matsumoto, Asymptopic series for double zeta, double gamma, and Hecke L-functions, Math. Proc. Camb. Phil. Soc. 123 (1998), 385-405; Corrigendum and addendum, ibid. 132 (2002), 377-384.

[8] K. Matsumoto, On analytic continuation of various multiple zeta-functions, in: M. A. Bennett et al.(Eds), Number Theory for the Mellennium II, Proc. Millennial Conference on Number Theory, A K Peters, Wellesley, 2002, pp. $417-440$.

[9] K. Matsumoto, Functional equation for double zeta-functions, Math. Proc. Cambridge Phil. Soc. 136 (2004), 1-7.

[10] L.J. Mordell, On the evaluation of some multiple series, J. London Math. Soc. 33 (1958), 368-371.

[11] T. Okamoto and T. Onozuka, Mean value theorems for the Mordell-Tornheim double zeta-function, Ramanujan J. 37 (2014), 131-163. 
[12] L. Tornheim, Harmonic double series, Amer. J. Math. 72 (1950), 303-314.

[13] E. Witten, On quantum gauge theories in two dimensions, Commun. Math. Phys. 141 (1991), 153-209.

[14] J. Zhao, Analytic continuation of multiple zeta functions, Proc. Amer. Math. Soc. 128 (2000), 1275-1283.

Addresses: Takuya Okamoto: Department of Mathematics, College of Liberal Arts and Sciences, Kitasato University, 1-15-1 Kitasato, Minami-ku, Sagamihara, Kanagawa 252-0373, Japan;

Tomokazu Onozuka: Graduate School of Mathematics, Nagoya University, Chikusa-ku, Nagoya 464-8602, Japan.

E-mail: takuyaok@kitasato-u.ac.jp, m11022v@math.nagoya-u.ac.jp

Received: 19 April 2015; revised: 14 April 2016 